\title{
Elaboración de aleaciones Cu-Al-Ni con efecto memoria de forma mediante pulvimetalurgia
}

\author{
R.B. Pérez-Sáez ${ }^{(*)}$, J. San Juan ${ }^{(*)}$, V. Recarte ${ }^{(*)}$, M.L. Nó $^{(* *)}$, G. Caruana $^{(* * *)}$ \\ y O.A. Ruano $(* * *)$
}

Resumen En la elaboración de aleaciones Cu-Al-Ni con efecto memoria de forma es importante conseguir un tamaño de grano fino, para mejorar las propiedades mecánicas y el comportamiento durante el ciclado termomecánico. Clásicamente, esto se ha conseguido mediante la adición de refinadores de grano; sin embargo, los efectos secundarios que éstos producen pueden ser problemáticos. Por esta razón, se ha desarrollado un nuevo método de procesado de este tipo de aleaciones mediante pulvimetalurgia. En este trabajo se presenta el proceso de elaboración consistente en tres etapas: atomización, compactación isostática en caliente y laminación en caliente. Se estudia la microestructura del material, se caracteriza la transformación martensítica y se determinan las propiedades termomecánicas.

Palabras clave: Memoria de forma. Pulvimetalurgia. Cu-Al-Ni. Transformación martensítica.

\section{Processing of $\mathrm{Cu}-\mathrm{Al}-\mathrm{Ni}$ shape memory alloys by powder metallurgy}

\begin{abstract}
During the production of shape memory alloys, a very fine grain size should be obtained in order to obtain better mechanical properties and a good thermomechanical behaviour during cycling. The classically used grain refiners show some secondary effects on the martensitic transformations that could be at the origin of some technological problems. For this reason, a new processing method by powder metallurgy has been developed for this kind of alloys. The three proceeding stages are described: Atomization, hot isostatic pressing and hot rolling. The microstructure of the materials is characterized. The martensitic transformation and the thermomechanical properties are also studied.
\end{abstract}

Keywords: Shape memory alloys. Powder metallurgy. Cu-Al-Ni. Martensitic transformation.

\section{INTRODUCCIÓN}

Las aleaciones con memoria (SMA) más utilizadas, $\mathrm{Cu}-\mathrm{Zn}-\mathrm{Al}$ y Ti-Ni, tienen la temperatura máxima de trabajo limitada a $120{ }^{\circ} \mathrm{C}$ debido a problemas de precipitación de las fases estables. A fin de elevar dicho límite, se han desarrollado otros sistemas de aleaciones, tales como Ni-Al, $\mathrm{Cu}-\mathrm{Ni}-\mathrm{Al}, \mathrm{Fe}-\mathrm{Mn}$ Si-X, Ti-Ni-Pd (1), cuyo límite está en torno a 240 ${ }^{\circ} \mathrm{C}$. Dado su bajo costo, facilidad de procesado y

(*) Dpto. Física Materia Condensada, Univ. País Vasco, Aptd. 644. 48080-Bilbao (España).

(**) Dpto. Física Aplicada II, Univ. País Vasco, Aptd. 644. 48080-Bilbao (España).

(***) Centro Nacional de Investigaciones Metalúrgicas, CENIM (CSIC), Avda. de Gregorio del Amo 8. 28040Madrid (España). buenas propiedades de memoria de forma, las aleaciones de $\mathrm{Cu}-\mathrm{Al}-\mathrm{Ni}$ son adecuadas para su utilización entre $100{ }^{\circ} \mathrm{C}$ y $240^{\circ} \mathrm{C}$. Sin embargo, debido a su alta anisotropía elástica $(\mathrm{A} \approx 13)$ y gran tamaño de grano, son frágiles y presentan pobres propiedades mecánicas. El método tradicional para obtener un tamaño de grano pequeño, con la consecuente mejora de propiedades mecánicas, es la adición de un cuarto elemento como afinador de grano, tales como el circonio, el titanio o el boro ( 2 y 3 ) que presentan, no obstante, el inconveniente de afectar al comportamiento de la transformación martensítica. Sin embargo, se han desarrollado métodos alternativos para obtener aleaciones con grano pequeño $(<100 \mu \mathrm{m})$, tales como la solidificación rápida (4-10) y la pulvimetalurgia (11-13). 
En el presente trabajo, se estudian las propiedades termomecánicas de una aleación $\mathrm{Cu}-\mathrm{Al}-\mathrm{Ni}$ con memoria de forma elaborada por un nuevo método pulvimetalúrgico.

\section{MÉTODO EXPERIMENTAL}

Partiendo de elementos de alta pureza $(\mathrm{Cu} 99,99$ $\%, \mathrm{Al} 99,99 \%$, Ni 99,97 \%) se ha obtenido, en un horno de inducción y en atmósfera inerte de argón, una aleación de composición nominal $\mathrm{Cu}-13 \mathrm{Al}-3 \mathrm{Ni}$ (\% en masa). En la figura 1 se muestra el esquema seguido durante la elaboración de la aleación. Primeramente, el fundido se ha atomizado mediante gas argón a 2,3 MPa de presión (14). El polvo obtenido fue analizado mediante ICP, obteniéndose una concentración de $\mathrm{Cu}-13,06 \mathrm{Al}-3,1 \mathrm{Ni}$ (\% en masa), siendo el tamaño medio de las partículas obtenidas de $90 \mu \mathrm{m}$. Para la compactación isostática en caliente (HIP), se usó el polvo, cuyo tamaño de par-

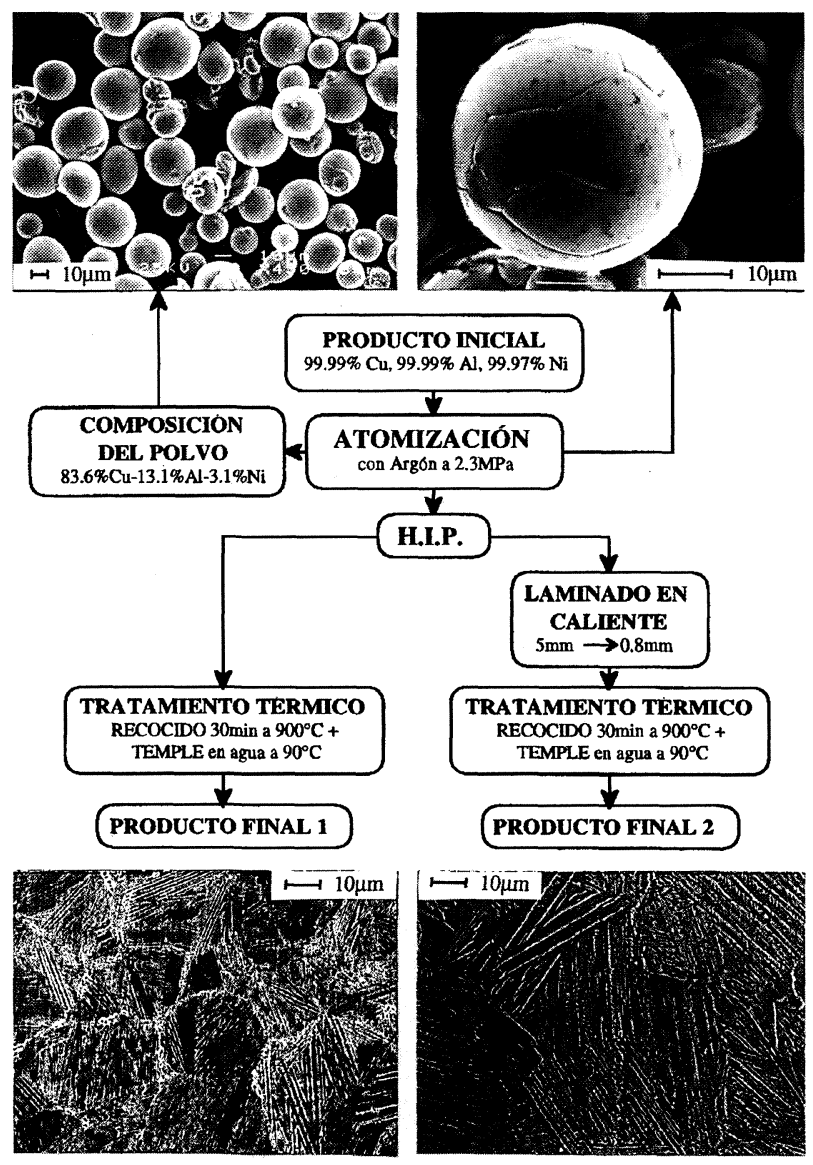

FIG. 1.- Esquema general del proceso de elaboración y micrografías correspondientes al polvo inicial y los productos finales.

FIG. 1.- General scheme of the elaboration process and microgaphs showing powders and final products microstructure. tícula estaba comprendido entre 50 y $25 \mu \mathrm{m}$. A partir de aquí, se han desarrollado dos procesos alternativos: Én el primero, la aleación consolidada por HIP se trata directamente (recocido de $30 \mathrm{~min}$ a 900 ${ }^{\circ} \mathrm{C}$ y temple en agua a $90{ }^{\circ} \mathrm{C}$ ); se obtiene el producto final 1 (PF1)(Fig. 1). En el segundo proceso, la muestra ha sido laminada en caliente previamente al mismo tratamiento térmico; se obtiene el producto final 2 (PF2) (Fig. 1).

Se ha caracterizado la transformación martensítica de ambos productos mediante fricción interna en un péndulo de torsión invertido que trabaja en el rango del $\mathrm{Hz}$ (15). Las temperaturas de transformación y la evolución de la fracción volumétrica se han obtenido a partir de la integral de la fricción interna (16). El estudio de las propiedades termomecánicas se ha realizado en una máquina de tracción-compresión dotada de un sistema de mordazas invertido que permite introducir la muestra en un baño termostático (17).

\section{MICROESTRUCTURA}

En la fotografía superior izquierda de la figura 1 se muestra la morfología de las partículas de polvo con tamaño entre 25 y $50 \mu \mathrm{m}$ utilizadas para la elaboración de la aleación. Se puede observar en la micrografía de la derecha que las partículas son policristalinas con un tamaño de grano entre 5 y 10 $\mu \mathrm{m}$.

En la fotografía inferior izquierda se muestra el producto final PF1. Se puede observar que el tamaño y la forma de los granos es semejante al de las partículas de partida debido a la presencia de una fina película de óxido intergranular que inhibe el crecimiento de grano. El laminado en caliente al que se ha sometido al producto PF2 rompe dicha película de óxido, obteniéndose un grano cuya forma no recuerda a la de las partículas de polvo y sin embargo se mantiene el tamaño de grano (micrografía inferior derecha).

\section{TRANSFORMACIÓN MARTENSÍTICA}

Las temperaturas de transformación obtenidas son: $M_{\mathrm{s}}=353 \mathrm{~K}, M_{\mathrm{f}}=294 \mathrm{~K}, A_{\mathrm{s}}=309 \mathrm{~K}, A_{\mathrm{f}}=369$ $\mathrm{K}$, para la muestra PF1 (Fig. $2 a$ ) y $M_{\mathrm{s}}=367 \mathrm{~K}$, $M_{\mathrm{f}}=322 \mathrm{~K}, A_{\mathrm{s}}=330 \mathrm{~K}$ y $A_{\mathrm{f}}=377 \mathrm{~K}$, para la muestra PF2 (Fig. 2b). La histéresis de transformación de la muestra PF1 $(\approx 13 \mathrm{~K})$ es ligeramente mayor que la histéresis de la muestra $\mathrm{PF} 2(\approx 8 \mathrm{~K})$, presentando ambas muestras una transformación del tipo $\beta=>\beta^{\prime}$. Las temperaturas de transformación de la muestra PF2 son ligeramente mayores que las de la PF1. Este efecto podría deberse a la restricción impuesta por la fina película de óxido 

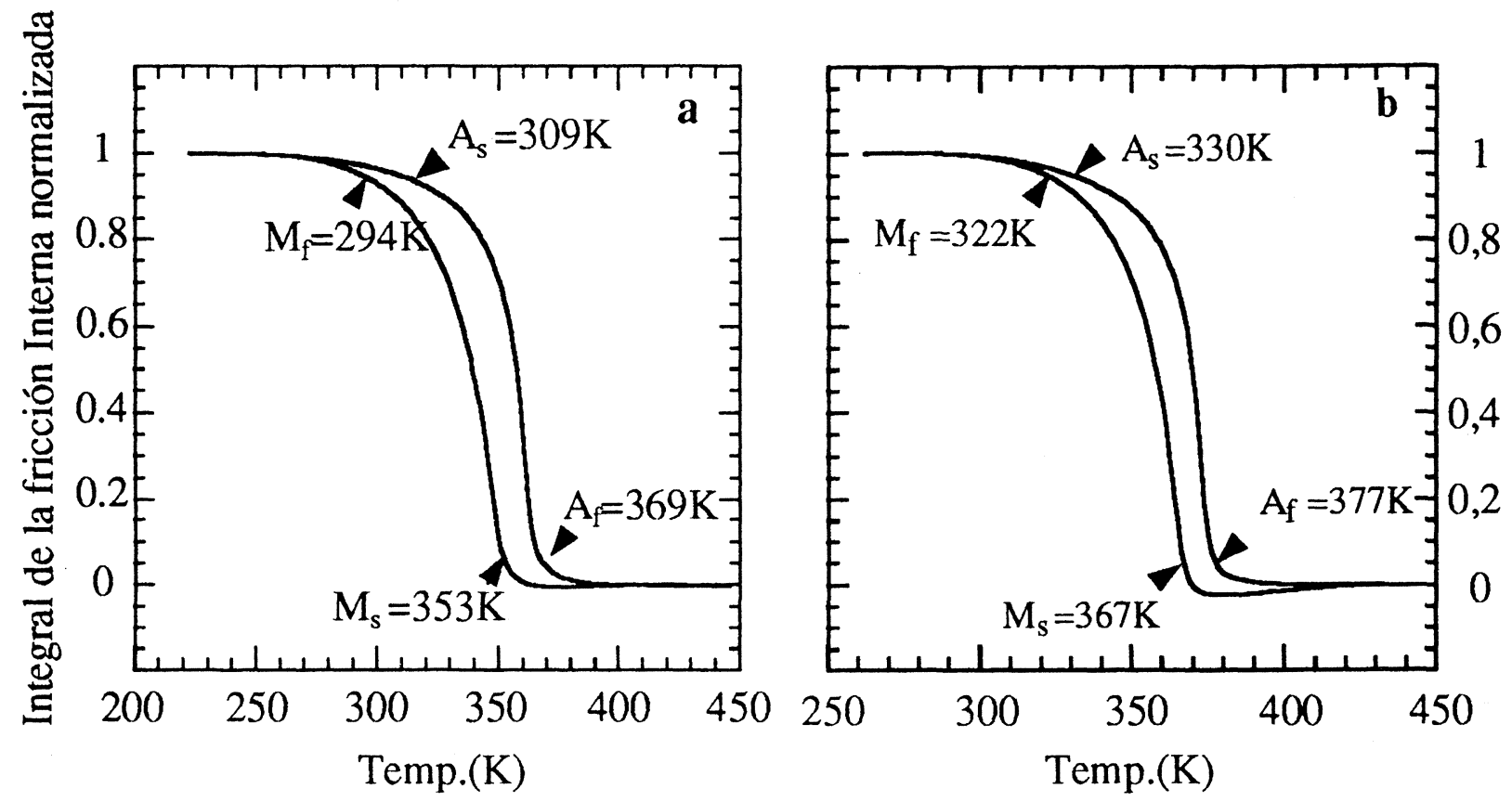

FIG. 2.- Integral normanalizada de la fricción interna. Temperaturas de transformación martensítica al 5\% y $95 \%$. a) muestra PF1, b) muestra PF2.

FIG. 2.- Normalized internal friction integral. Martensitic transformation temperatures at 5\% and 95\%. a) $P F 1, b) P F 2$.

intergranular presente en la muestra tras el HIP y que es eliminado en el posterior laminado en caliente. Esta interpretación concuerda con el hecho de que la transformación martensítica que presentan las partícula de polvo libres de tensiones tiene lugar a temperaturas notablemente más bajas que PF1 y PF2 (18).

\section{PROPIEDADES TERMOMECÁNICAS}

Se ha estudiado el comportamiento superelástico mediante ensayos hasta el $1 \%$ de deformación a temperaturas superiores a $A_{\mathrm{f}}$. En dicho rango de temperaturas, la muestra está en fase $\beta$ y la transformación martensítica es inducida por tensión, incrementándose la tensión necesaria para inducir dicha transformación al aumentar la temperatura. La relación obtenida entre la tensión de transformación y la temperatura es lineal para ambas muestras (Fig. 3), cumpliéndose una relación de ClausiusClapeyron. Por otra parte, la pendiente de la muestra PF1 es mayor que la de la muestra PF2, debido a las tensiones internas generadas por la película de óxido intergranular presente en PF1 y que se opone a la transformación, siendo necesario incrementar la tensión aplicada para inducir la transformación. Igualmente, se han realizado ensayos superelásticos sobre la muestra PF2 a $393 \mathrm{~K}$ hasta el 4,5\% de deformación, obteniéndose un buen comportamien-

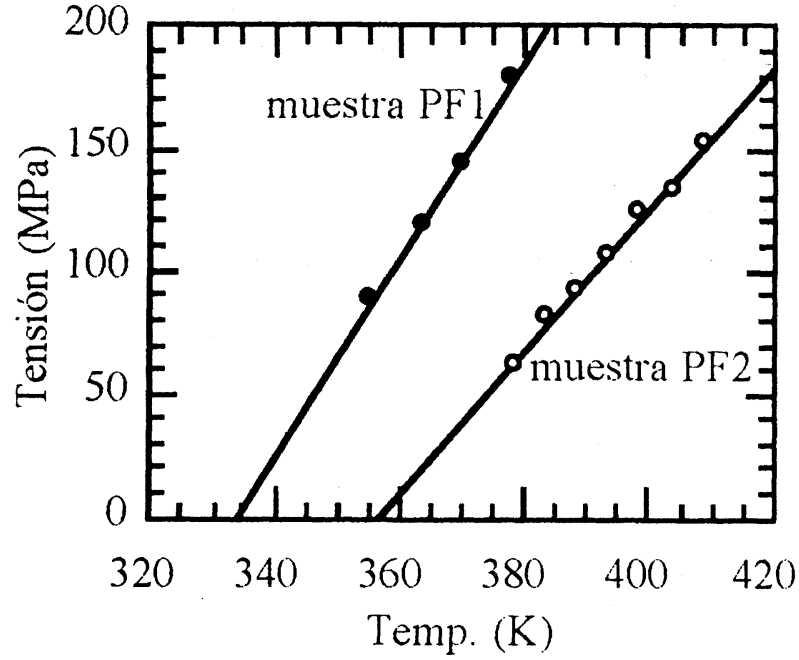

FIg. 3.- Relación de Claussius-Clapeyron para las muestras PF1 y PF2.

FIG. 3.-Claussius-Clapeyron relationship for PF1 and PF2 samples.

to mecánico de la misma. Sin embargo, la muestra $\mathrm{PF} 1$ en ensayos a $368 \mathrm{~K}$ presenta fractura integranular cuando se supera el $2 \%$ de deformación.

Con el fin de el estudiar el efecto de memoria simple, sobre la muestra PF2 se han realizado ensayos hasta el $7 \%$ de deformación a $277 \mathrm{~K}$, temperatura por debajo de $M_{\mathrm{f}}$, recuperándose la deformación por calentamiento libre de carga hasta 
temperaturas por encima de $A_{\mathrm{f}}$ (Fig. 4). Cuando retiramos la tensión, se recupera una deformación pseudoelástica del $2,75 \%$, quedando una deformación residual del 4,25\% (Fig. 4 a). Esta deformación se recupera por efecto memoria de forma simple durante el calentamiento por encima de $A_{\mathrm{f}}$, (Fig. $4 b$ ). Un enfriamiento posterior nos permite recuperar la forma en fase martensita. Por tanto, la muestra recupera el $88 \%$ de deformación por efecto de memoria simple durante el primer ciclo.

\section{CONCLUSIONES}

El proceso de elaboración mediante pulvimetalurgia se ha mostrado adecuado para obtener aleaciones con memoria de forma de grano fino. La muestra laminada en caliente PF2 muestra mejores propiedades termomecánicas que la muestra sin laminar PF1. Cabe resaltar que la muestra PF2 pre-
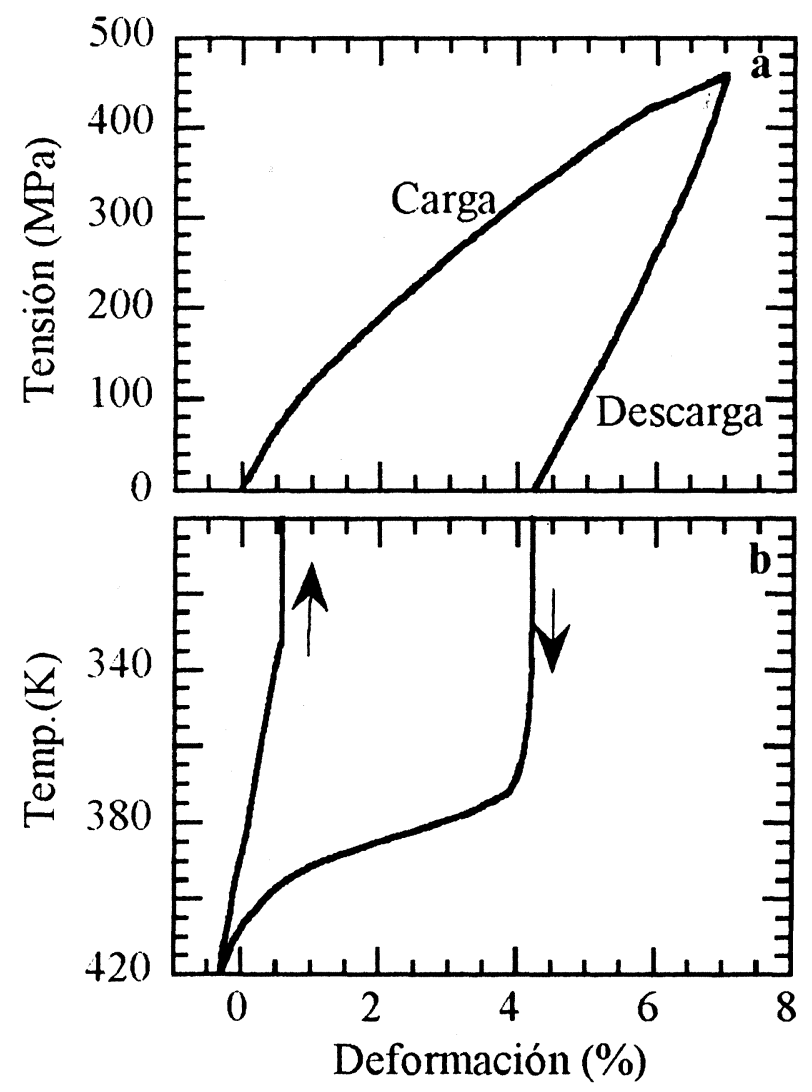

Fig. 4.- a) Curva tensión-deformación para la muestra PF2 a 277 K. b) Curva de recuperación de la deformación aplicada en la fig. 4-a.

FIG. 4.- a) Tensile stress-strain curve until 7\% deformation for the PF2 sample at $277 \mathrm{~K} . b$ ) Shape memory recovery curve after unloading in fig. 4-a. senta propiedades termomecánicas superiores a las de las aleaciones $\mathrm{Cu}-\mathrm{Al}-\mathrm{Ni}$ obtenidas por métodos convencionales.

\section{Agradecimiento}

El presente trabajo ha sido realizado gracias a la financiación de la CICYT en el marco del Plan Nacional de Materiales (Proyecto MAT92-0353) y de la Universidad del País Vasco (Proyecto UPV 063.310-EB049195).

\section{REFERENCIAS}

(1) Delaey, L. "Diffusionless Transformation" en Phase Transformation in Materials. P. Haasen Ed., VCH (Weinheim, Alemania), 1991: 339-404.

(2) San Juan, J., Recarte, V., Herreros, J., Hurtado, I., Nó, M.L. Proc Conf. Nac. Ciencia y Tecnología Metalúrgicas, CENIM. Madrid (España) 3-5 Oct. 1990. Vol.II: 71-80.

(3) Récarte, V. Tesis Doctoral, U.P.V., Bilbao (España).1997.

(4) Stobis, W.M. y Wood, J.V. Acta Metall. 27, 1979: 575584.

(5) Wood, J.V. J. Physique C4 43, 1982: 755-760.

(6) Oshima, R., Tanimoto, M., Oka, T., Fujita, F.E., Hanadate, Y., Hamada, T. y Miyagi, M. J. Physique C4. 43, 1982: 749-754.

(7) Wood, J.V. y Shingu, P.H. Metall. Trans. A 15, 1984: 471-480.

(8) Ahmed, M., Husain, S.W., IQbal, Z., Hashmi, F.H. y KHAN, A.Q. Scr. Metall. 22, 1988: 803-808.

(9) Zhu, J.H., Dunne, D.P., Delamore, G.W. y Kennon, N.F. Proc. Int. Conf. Martensitic Transformations, Monterey (Ca, EE.UU.) 20-24 Jul. 1992, (C.M. Wayman y J. Perkins, Eds.) Inst. for Advanced Studies, Monterey, 1993: $1.083-1.088$

(10) Donner, P. y Eucken, S. Mater. Sci. Forum 58, 1990: 723.

(11) Duering, T.W., Albrecht, J. y Gessinger, G.H. J. Metals 43, 1982: 14-20.

(12) Jassen, J., Willems, F., Verelst, B., Maertens, J. y Delaey, L. J. Physique C4 34,1982: 809-812.

(13) JEAn, R.D., Wu, T.Y. y Leu, S.S. Scr. Metall. 25, 1991: 883-888.

(14) Lieblich, M., Caruana, G., Torralba, M. y Jones, H. Mater. Sci. Techn. 12, 1996: 25

(15) Oleaga, A., Hurtado, I., Nó, M.L., Snouf, C. y San JuAN, J. An. Fís. 85B, 1989: 375

(16) Pérez-Saez, R.B., Recarte, V., No, M.L. y San Juan, J. Phys. Rev. B. 1998 (En prensa).

(17 Pérez-SAez, R.B. Tesis Doctoral, Univ. del País Vasco. Bilbao (España) 1998.

(18) San Juan, J., Pérez-Saez, R.B., Recarte, V., No, M.L., Caruana, G., Lieblich, M. y Ruano, O.A. J. Physique IV, C8-919 (1995). 